\title{
Sudden stratospheric warmings seen in MINOS deep underground muon data
}

\author{
S. Osprey \\ J. Barnett \\ J. Smith \\ S. J. Coleman \\ William \& Mary, racole@wm.edu \\ M. Kordosky \\ William \& Mary, makordosky@wm.edu \\ See next page for additional authors
}

Follow this and additional works at: https://scholarworks.wm.edu/aspubs

\section{Recommended Citation}

Osprey, S., Barnett, J., Smith, J., Adamson, P., Andreopoulos, C., Arms, K. E., ... \& Barnes, P. D. (2009). Sudden stratospheric warmings seen in MINOS deep underground muon data. Geophysical Research Letters, 36(5). accepted for inclusion in Arts \& Sciences Articles by an authorized administrator of W\&M ScholarWorks. For more information, please contact scholarworks@wm.edu. 
Authors

S. Osprey, J. Barnett, J. Smith, S. J. Coleman, M. Kordosky, J. K. Nelson, and P. Vahle 


\title{
Sudden stratospheric warmings seen in MINOS deep underground muon data
}

\author{
S. Osprey, ${ }^{1, *}$ J. Barnett, ${ }^{1}$ J. Smith, ${ }^{1}$ P. Adamson, ${ }^{2, \dagger}$ C. Andreopoulos, ${ }^{3}$ K. E. Arms, ${ }^{4}$
} R. Armstrong, ${ }^{5}$ D. J. Auty, ${ }^{6}$ D. S. Ayres, ${ }^{7}$ B. Baller, ${ }^{2}$ P. D. Barnes Jr., ${ }^{8}$ G. D. Barr, ${ }^{1}$ W. L. Barrett, ${ }^{9}$ B. R. Becker, ${ }^{4}$ A. Belias, ${ }^{3}$ R. H. Bernstein, ${ }^{2}$ D. Bhattacharya, ${ }^{10}$ M. Bishai, ${ }^{11}$ A. Blake, ${ }^{12}$ G. J. Bock, ${ }^{2}$ J. Boehm, ${ }^{13}$ D. J. Boehnlein, ${ }^{2}$ D. Bogert, ${ }^{2}$ C. Bower, ${ }^{5}$ E. Buckley-Geer, ${ }^{2}$ S. Cavanaugh, ${ }^{13}$ J. D. Chapman, ${ }^{12}$ D. Cherdack, ${ }^{14}$ S. Childress, ${ }^{2}$ B. C. Choudhary, ${ }^{2}$ J. H. Cobb, ${ }^{1}$ S. J. Coleman, ${ }^{15}$ A. J. Culling, ${ }^{12}$ J. K. de Jong, ${ }^{16}$ M. Dierckxsens, ${ }^{11}$ M. V. Diwan, ${ }^{11}$ M. Dorman, ${ }^{17,18}$ S. A. Dytman, ${ }^{10}$ C. O. Escobar, ${ }^{19}$ J. J. Evans, ${ }^{1,20}$ E. Falk, ${ }^{6}$ G. J. Feldman, ${ }^{13}$ M. V. Frohne, ${ }^{21}$ H. R. Gallagher, ${ }^{14}$ A. Godley, ${ }^{22}$ M. C. Goodman, ${ }^{7}$ P. Gouffon, ${ }^{23}$ R. Gran, ${ }^{24}$ E. W. Grashorn, ${ }^{4,25}$ N. Grossman, ${ }^{2}$ K. Grzelak, ${ }^{26,27}$ A. Habig, ${ }^{24}$ D. Harris, ${ }^{2}$ P. G. Harris, ${ }^{6}$ J. Hartnell, ${ }^{6,18}$ R. Hatcher, ${ }^{2}$ A. Himmel, ${ }^{28}$ A. Holin, ${ }^{17}$ J. Hylen, ${ }^{2}$ G. M. Irwin, ${ }^{29}$ M. Ishitsuka, ${ }^{5}$ D. E. Jaffe, ${ }^{11}$ C. James, ${ }^{2}$ D. Jensen, ${ }^{2}$ T. Kafka, ${ }^{14}$ S. M. S. Kasahara, ${ }^{4}$ J. J. Kim, ${ }^{22}$ G. Koizumi, ${ }^{2}$ S. Kopp ${ }^{30}$ M. Kordosky, ${ }^{15,31}$ D. J. Koskinen, ${ }^{17}$ A. Kreymer, ${ }^{2}$ S. Kumaratunga, ${ }^{4}$ K. Lang, ${ }^{30}$ J. Ling, ${ }^{22}$ P. J. Litchfield, ${ }^{4}$ R. P. Litchfield, ${ }^{1}$ L. Loiacono, ${ }^{30}$ P. Lucas, ${ }^{2}$ J. Ma, ${ }^{30}$ W. A. Mann, ${ }^{14}$ M. L. Marshak, ${ }^{4}$ J. S. Marshall, ${ }^{12}$ N. Mayer, ${ }^{5}$ A. M. McGowan, ${ }^{7,32}$ J. R. Meier, ${ }^{4}$ M. D. Messier, ${ }^{5}$ C. J. Metelko, ${ }^{3}$ D. G. Michael, ${ }^{28,33}$ L. Miller, ${ }^{34,35}$ W. H. Miller, ${ }^{4}$ S. R. Mishra, ${ }^{22}$ C. D. Moore, ${ }^{2}$ J. G. Morfin, ${ }^{2}$ L. Mualem, ${ }^{28}$ S. Mufson, ${ }^{36}$ J. Musser, ${ }^{5}$ D. Naples, ${ }^{10}$ J. K. Nelson, ${ }^{15}$ H. B. Newman, ${ }^{28}$ R. J. Nichol, ${ }^{17}$ T. C. Nicholls, ${ }^{3}$ J. P. Ochoa-Ricoux, ${ }^{28}$ W. P. Oliver, ${ }^{14}$ R. Ospanov, ${ }^{30}$ J. Paley, ${ }^{5}$ V. Paolone, ${ }^{10}$ Z. Pavlovic, ${ }^{30}$ G. Pawloski, ${ }^{29}$ G. F. Pearce, ${ }^{3}$ C. W. Peck, ${ }^{28}$ D. A. Petyt,${ }^{4}$ R. Pittam, ${ }^{1}$ R. K. Plunkett, ${ }^{2}$ A. Rahaman, ${ }^{22}$ R. A. Rameika, ${ }^{2}$ T. M. Raufer, ${ }^{3}$ B. Rebel, ${ }^{2}$ J. Reichenbacher, ${ }^{7}$ P. A. Rodrigues, ${ }^{1}$ C. Rosenfeld ${ }^{22}$ H. A. Rubin, ${ }^{16}$ K. Ruddick, ${ }^{4}$

\footnotetext{
${ }^{1}$ Department of Physics, University of Oxford, Oxford, UK.

${ }^{2}$ Fermi National Accelerator Laboratory, Batavia, Illinois, USA.

${ }^{3}$ Rutherford Appleton Laboratory, Chilton, UK.

${ }^{4}$ School of Physics and Astronomy, University of Minnesota-Twin Cities, Minneapolis, Minnesota, USA.

${ }^{5}$ Department of Physics, Indiana University, Bloomington, Indiana, USA. UK.

${ }^{6}$ Department of Physics and Astronomy, University of Sussex, Falmer,

${ }^{7}$ Argonne National Laboratory, Argonne, Illinois, USA USA.

${ }^{8}$ Lawrence Livermore National Laboratory, Livermore, California,

${ }^{9}$ Physics Department, Western Washington University, Bellingham, Washington, USA.

${ }^{10}$ Department of Physics and Astronomy, University of Pittsburgh, Pittsburgh, Pennsylvania, USA.

${ }^{11}$ Brookhaven National Laboratory, Upton, New York, USA.

${ }^{12}$ Cavendish Laboratory, University of Cambridge, Cambridge, UK.

${ }^{13}$ Department of Physics, Harvard University, Cambridge, Massachusetts, USA.

${ }^{14}$ Physics Department, Tufts University, Medford, Massachusetts, USA.

${ }^{15}$ Department of Physics, College of William and Mary, Williamsburg, Virginia, USA.

${ }^{16}$ Physics Division, Illinois Institute of Technology, Chicago, Illinois, USA.

${ }^{17}$ Department of Physics and Astronomy, University College London, London, UK.

${ }^{18}$ Formerly at Rutherford Appleton Laboratory, Chilton, UK.

${ }^{19}$ Instituto de Fsica Gleb Wataghin, Universidade Estadual de Campinas, Campinas, Brazil.

${ }^{20}$ Now at Department of Physics and Astronomy, University College London, London, UK.

${ }^{21}$ Physics Department, Benedictine University, Lisle, Illinois, USA.
}

\footnotetext{
${ }^{22}$ Department of Physics and Astronomy, University of South Carolina, Columbia, South Carolina, USA.

${ }^{23}$ Instituto de Fisica, Universidade de Sao Paulo, Sao Paulo, Brazil.

${ }^{24}$ Department of Physics, University of Minnesota at Duluth, Duluth, Minnesota, USA

${ }^{25}$ Formerly at Department of Physics, University of Minnesota at Duluth, Duluth, Minnesota, USA.

${ }^{26}$ Faculty of Physics, Warsaw University, Warsaw, Poland.

${ }^{27}$ Formerly at Department of Physics, University of Oxford, Oxford, UK.

${ }^{28}$ Lauritsen Laboratory, California Institute of Technology, Pasadena, California, USA.

${ }^{29}$ Department of Physics, Stanford University, Stanford, California, USA. USA.

${ }^{30}$ Department of Physics, University of Texas at Austin, Austin, Texas,

${ }^{31}$ Formerly at Department of Physics and Astronomy, University College London, London, UK.

${ }^{32}$ Formerly at School of Physics and Astronomy, University of Minnesota-Twin Cities, Minneapolis, Minnesota, USA.

${ }^{33}$ Deceased 25 December 2005.

${ }^{34}$ Physics Department, James Madison University, Harrisonburg, Virginia, USA.

${ }^{35}$ Deceased 16 May 2001.

${ }^{36}$ Department of Astronomy, Indiana University, Bloomington, Indiana, USA.

${ }^{37}$ Department of Physics, University of Athens, Athens, Greece.

${ }^{38}$ Deceased 16 August 2004. USA.

${ }^{39}$ Physics Department, Texas A\&M University, College Station, Texas,

*This work was carried out by physicists from the National Centre for Atmospheric Science (NCAS) and the MINOS collaboration. The NCAS authors are listed, followed by the MINOS authors.

$\dagger$ The MINOS authors are listed from this point. The authors are arranged alphabetically by surname.
} 
M. C. Sanchez,${ }^{7,13}$ N. Saoulidou, ${ }^{2}$ J. Schneps, ${ }^{14}$ P. Schreiner, ${ }^{21}$ S. M. Seun, ${ }^{13}$ P. Shanahan, ${ }^{2}$ W. Smart, ${ }^{2}$ C. Smith, ${ }^{17}$ R. Smith, ${ }^{1}$ A. Sousa, ${ }^{1}$ B. Speakman, ${ }^{4}$ P. Stamoulis, ${ }^{37}$ M. Strait, ${ }^{4}$ P. Symes, ${ }^{6}$ N. Tagg, ${ }^{14}$ R. L. Talaga, ${ }^{7}$ M. A. Tavera, ${ }^{6}$ J. Thomas, ${ }^{17}$ J. Thompson, ${ }^{10,38}$ M. A. Thomson, ${ }^{12}$ J. L. Thron, ${ }^{7}$ G. Tinti, ${ }^{1}$ G. Tzanakos, ${ }^{37}$ J. Urheim, ${ }^{5}$ P. Vahle, ${ }^{15,31}$ B. Viren, ${ }^{11}$ M. Watabe, ${ }^{39}$ A. Weber, ${ }^{1}$ R. C. Webb, ${ }^{39}$ A. Wehmann, ${ }^{2}$ N. West, ${ }^{1}$ C. White, ${ }^{16}$ S. G. Wojcicki, ${ }^{29}$ D. M. Wright, ${ }^{8}$ T. Yang, ${ }^{29}$ K. Zhang, ${ }^{11}$ and R. Zwaska ${ }^{2}$

Received 16 October 2008; accepted 15 January 2009; published 7 March 2009.

[1] The rate of high energy cosmic ray muons as measured underground is shown to be strongly correlated with upperair temperatures during short-term atmospheric (10-day) events. The effects are seen by correlating data from the MINOS underground detector and temperatures from the European Centre for Medium Range Weather Forecasts during the winter periods from 2003-2007. This effect provides an independent technique for the measurement of meteorological conditions and presents a unique opportunity to measure both short and long-term changes in this important part of the atmosphere. Citation: Osprey, S., et al. (2009), Sudden stratospheric warmings seen in MINOS deep underground muon data, Geophys. Res. Lett., 36, L05809, doi:10.1029/2008GL036359.

\section{Introduction}

[2] Primary cosmic rays, consisting of protons and other nuclei, are constantly entering the solar system producing a flux of around $70 \mathrm{~m}^{-2} \mathrm{sr}^{-1} \mathrm{~s}^{-1}$ for particles above $1 \mathrm{GeV}$ at the top of the atmosphere [Gaisser, 1990]. The subsequent interactions in the atmosphere (peaking at an altitude of about $15 \mathrm{~km}$ ) produce short-lived mesons (charged pions and kaons) which can then decay, producing muons. Muons are penetrating particles with a relatively long lifetime and can be observed on the Earth's surface and deep underground. The minimum muon energy required to reach $705 \mathrm{~m}$ underground, the location of the MINOS detector used in this study, is $0.7 \mathrm{TeV}$.

[3] There are two mechanisms by which the condition of the atmosphere affects the muon rate. Firstly, an increase in temperature causes the atmosphere to expand so muons are produced higher up and therefore have a larger probability to decay before being detected. Secondly, the mesons may interact (and thereby be lost) as well as decay. As the temperature increases, the probability of interaction becomes smaller because the local atmospheric density decreases, so more mesons decay, causing an increase in the muon rate. In deep underground detectors where muons with a high surface energy are measured, the second effect dominates and this causes a positive correlation between temperature and muon rate.This was first seen in a seasonal variation of the muon rate [Ambrosio et al., 1997].

[4] In the atmosphere, planetary Rossby waves can have horizontal wavelengths of several thousand kilometers [Andrews et al., 1987]. These waves propagate up from the troposphere to the stratosphere during winter [Charney and Drazin, 1961] and have an association with highlatitude vortex structures in the upper atmosphere. In analogy to water waves these waves can break causing temperatures in the polar stratosphere to rise by over $50 \mathrm{~K}$ in a few days. These events are known as Sudden Stratospheric
Warmings (SSW) and appear as a displacement or splitting of a large persistent low pressure system which resides over the pole, known as the wintertime stratospheric polar vortex. These events are observed as anomalous changes in temperature and wind at high latitudes. The frequency of SSW are thought to be tied loosely to the wind direction in the tropical stratosphere [Holton and Tan, 1980] and seasurface temperatures [Chen et al., 2003]. The distribution of the major land-masses provides a means of generating these waves, as air is pushed up as it moves over steep terrain. Consequently most recorded instances of SSW have occurred in the Northern Hemisphere where most land surface is found, with only one recorded instance in the Southern Hemisphere [Varotsos, 2002].

[5] SSW were discovered in the early 1950s by Scherhag using radiosonde measurements over Berlin [Scherhag, 1952]. Since then, radiosonde measurements have provided the mainstay in recording meteorological data in the upper troposphere and lower stratosphere. However, rocket soundings, LIDAR, airborne observatories and satellite measurements have increasingly improved our understanding of the upper atmosphere and phenomena such as SSW. This paper provides the first observational evidence of a SSW in the Northern Hemisphere using secondary cosmic ray muons deep underground.

\section{MINOS Far Detector}

[6] For this measurement, the MINOS neutrino detector was used to detect muons [Adamson et al., 2007]. The 5400 tonne detector (D. G. Michael et al., manuscript in preparation, 2009) consists of 486 vertical magnetised steel sheets interleaved with plastic scintillator detector planes, which are regular octagons, $8 \mathrm{~m}$ from side-to-side. The detector is $29 \mathrm{~m}$ long. The scintillator planes are segmented into $4.1 \mathrm{~cm}$ strips in alternating perpendicular directions to allow particle tracks to be viewed in two orthogonal $2 \mathrm{D}$ views. The passage of muons is detected with photomultiplier tubes and both pulse height and arrival times are digitised. Pattern recognition software is used to reconstruct tracks, first in 2D and then 3D. Tracks are required to pass through at least five of the $2.56 \mathrm{~cm}$ thick steel planes (a threshold local muon energy of $200 \mathrm{MeV}$ ) to remove the chance of noise or radioactively induced hits accidentally forming a track. High efficiency and therefore a high stability for counting muons is achieved by designing the reconstruction software to tolerate missing hits along the track. This results in a total acceptance for the MINOS Far Detector to single atmospheric muons of $\left(1 \times 10^{7}\right) \mathrm{cm}^{2}$ sr [Adamson et al., 2007; Rebel, 2004] for the above data selection. The detector has been operational from August 2003 to the present with a live-time percentage in the time period analysed in this paper of $93.4 \%$. 


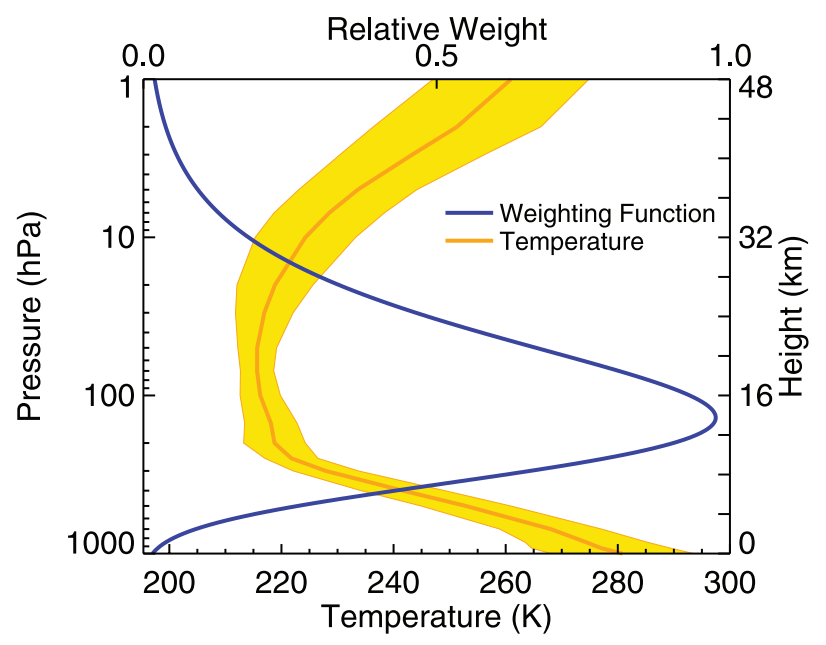

Figure 1. Muon Weighting Function. Scaling factor used to determine effective temperature (blue, top axis, arbitrary scale) and climatological temperature \pm 1 standard deviation for the period 2003-2007 from ECMWF (solid yellow, bottom axis).

[7] Upper-air temperature data were provided by the British Atmospheric Data Centre (BADC). This comprised the 4-daily Operational Analysis data from the European Centre for Medium-Range Weather Forecasts (ECMWF). These data combine a range of environmental data in a meteorologically consistent fashion using a process known as data assimilation [Courtier et al., 1994; ECMWF, 2007]. An added advantage of these data is that they are contiguous both spatially and in time. For temperature data, these comprise (interpolated) $1^{\circ}$ by $1^{\circ}$ globally gridded data and provide 21 discrete pressure levels extending from the surface to $1 \mathrm{hPa}$ (about $48 \mathrm{~km}$ ) altitude.

[8] Following Barrett et al. [1952] and Ambrosio et al. [1997], a single combined effective temperature $T$ is defined by making a weighted average temperature as a function of altitude as shown in Figure 1. The weighting is given by the distribution of altitudes where the mesons which cause observed muons in MINOS occur, based on analytic calculation [Ambrosio et al., 1997; Gaisser, 1990]. Figure 1 shows that most of the mesons are located about the tropopause $(\sim 100 \mathrm{hPa})$ where temperature is least variable. However, some mesons exist in the troposphere (below $\sim 300 \mathrm{hPa}$ ), where baroclinic weather systems are associated with most of the variability. Mesons also occur in the mid-stratosphere (above $\sim 10 \mathrm{hPa}$ ) which experiences variability (especially during wintertime) due to a more pronounced annual cycle than at lower altitudes, the presence of large-scale waves and decreasing air density. The average over the 21 pressure levels, using weights shown in Figure 1, gives the effective temperature $T$. To obtain data suitable for this study, a linear interpolation was performed (in longitude and latitude) using the ECMWF temperatures nearest the MINOS Far Detector.

\section{Results}

[9] The position of the polar vortex on February 6, 2005 is shown in Figure 2 which is at a time of low temperatures. Also shown is the location of the MINOS detector, in the town of Soudan, Minnesota, USA at lat. $47.8^{\circ} \mathrm{N}$, lon. $92.2^{\circ} \mathrm{W}$. Ertel's Potential Vorticity, usefully conserved over a period of several days in the stratosphere, is a measure of the location, size and shape of the winter polar vortex, is also shown in Figure 2. During this time, part of the polar vortex covers the MINOS site. The presence of the polar cyclone brings with it cooler temperatures and relatively strong westerly winds (not shown). The shape of the vortex is elliptical which suggests that large-scale Rossby waves are present and propagating up into the stratosphere. These waves are linked intimately with the Aleutian High (a region of high pressure located about the North Pacific). The region of high temperature seen in Figure 2 identifies the position of this anticyclone. By February 16, the shape of the polar vortex has changed and no longer extends over Soudan (Figure 2, bottom). Furthermore, the Aleutian High has broadened and reaches longitudes near Soudan. This behaviour is suggestive of a particular SSW event, where the Aleutian High strengthens and displaces a weakened polar vortex from the pole [Andrews et al., 1987].

[10] The variation in muon rate $R$ measured in MINOS for the winter 2004-2005 and the corresponding effective temperature $T$ are shown as a function of time in Figure $3 \mathrm{~b}$. To better highlight covariations in muon rate and temperature, a 5-day rolling average is applied twice to each timeseries. The correlation of the daily unfiltered data is 0.90 with a regression coefficient $\alpha=(T / R) \mathrm{d} R / \mathrm{d} T$ of $0.87 \pm$ 0.03 . This value for $\alpha$ compares well with expectations from a model of pion production and decay which predicts $\alpha=0.91$ at MINOS depths [Ambrosio et al., 1997]. This model gives an overestimate of $\alpha$ because kaons are neglected (P. Adamson et al., manuscript in preparation, 2009). During late autumn and throughout winter, the effective temperature gradually decreases before reaching a minimum in early February. The boreal winter of 20042005 had the lowest temperatures on record in the polar stratosphere [Manney et al., 2006; Rex et al., 2006]. Linked with this, ozone concentrations in the polar vortex were anomalously low, and the amounts of polar stratospheric clouds and chemically active chlorine were high [Tilmes et al., 2006]. Throughout this time, variations in both effective temperature and muon flux are highly correlated. A conspicuous feature in both muon flux and temperature is seen in late winter (February); both show a sharp rise followed by a fall over a period of $2-3$ weeks during which the muon rate changes by $(4 \pm 1) \%$. This behaviour is consistent with Rossby wave activity which has been associated with a split in the vortex in the lower stratosphere [Chshyolkova et al., 2007]. Following this warming and cooling, temperatures and muon rate are seen to increase once more, coinciding with an early final warming (and break-up) of the vortex on March 10, with the transition from winter to summer conditions. It is suggested, that had polar temperatures not risen due to the early break up of the winter vortex, greater ozone destruction may have occurred [Feng et al., 2007].

[11] The muon flux variation measured by MINOS and the corresponding effective temperature are also shown for the preceding (Figure 3a) and subsequent two winters (Figures $3 \mathrm{c}$ and $3 \mathrm{~d}$ ). All years show a similarly strong correlation between $R$ and $T$. Similarly to the winter of 


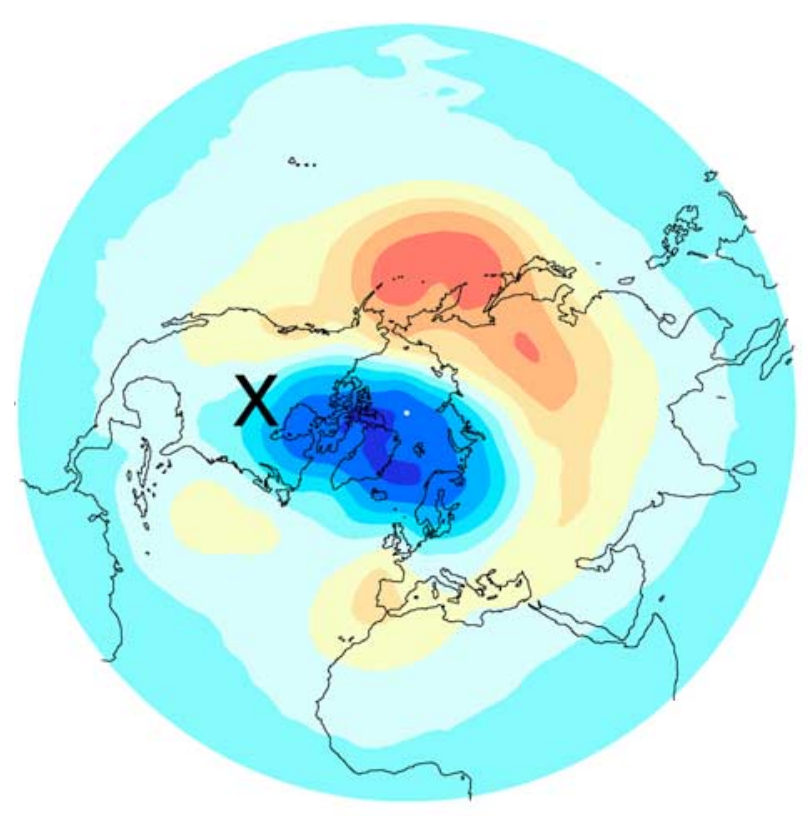

203206209212215218221224227230233236 Effective Temperature (K)

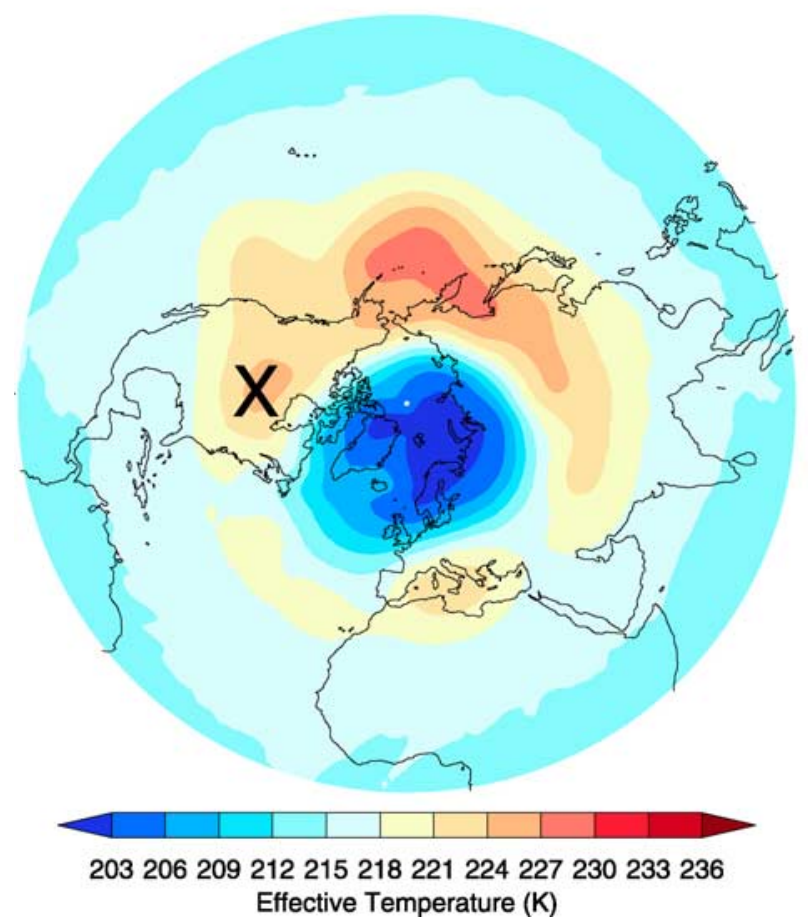

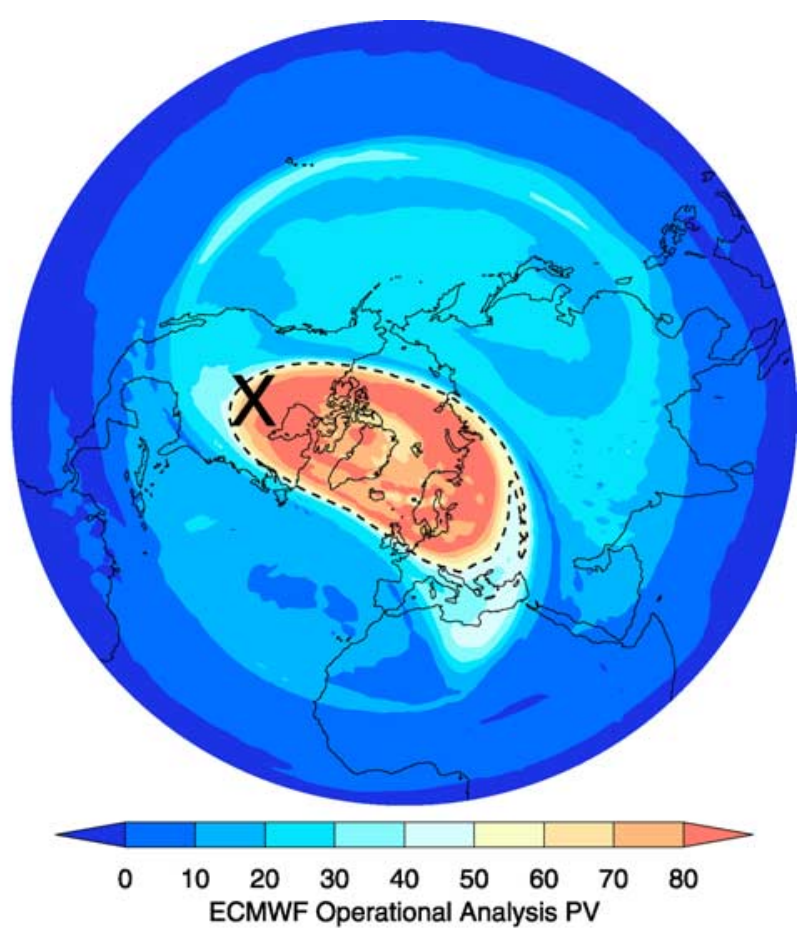

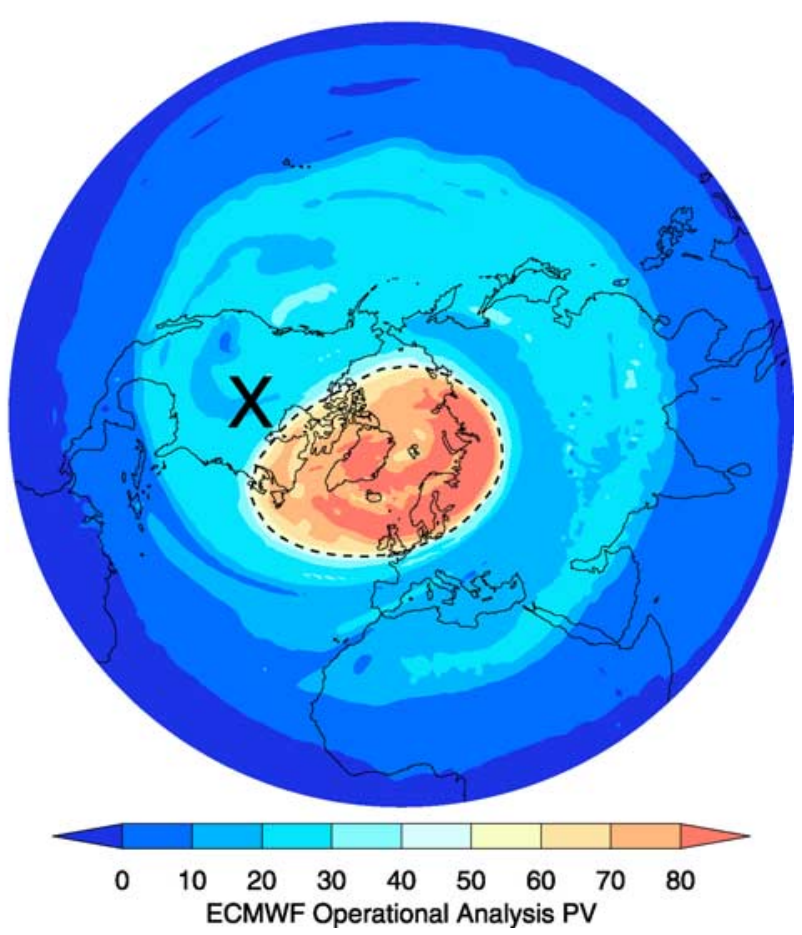

Figure 2. (left) Effective temperature and (right) potential vorticity at $850 \mathrm{~K}$ for (top) 6 and (bottom) 16 February 2005 derived from ECMWF. Units are $\mathrm{K}$ and $10^{-4} \mathrm{~K} \mathrm{~m}^{2} \mathrm{~kg}^{-1} \mathrm{~s}^{-1}$, respectively. The location of Soudan is marked by an $X$.

2004/2005, the other winter periods show times of increased temperature and muon flux most likely associated with the presence of Rossby waves (A. Charlton-Perez, personal communication, 2007).

\section{Discussion}

[12] For the first time, daily variations in secondary cosmic rays from an underground muon detector are shown to be associated with planetary-scale meteorological phenomena in the stratosphere. These phenomena occur over timescales from around ten days to two weeks and are intimately linked with the wintertime stratospheric polar vortex. The correspondence with the MINOS data is striking, especially during the winter months. As a significant fraction of tropospheric ionisation is thought to be produced by secondary cosmic muon radiation [Aplin et al., 2005; Bazilevskaya et al., 2008; Sloan and Wolfendale, 2008], 

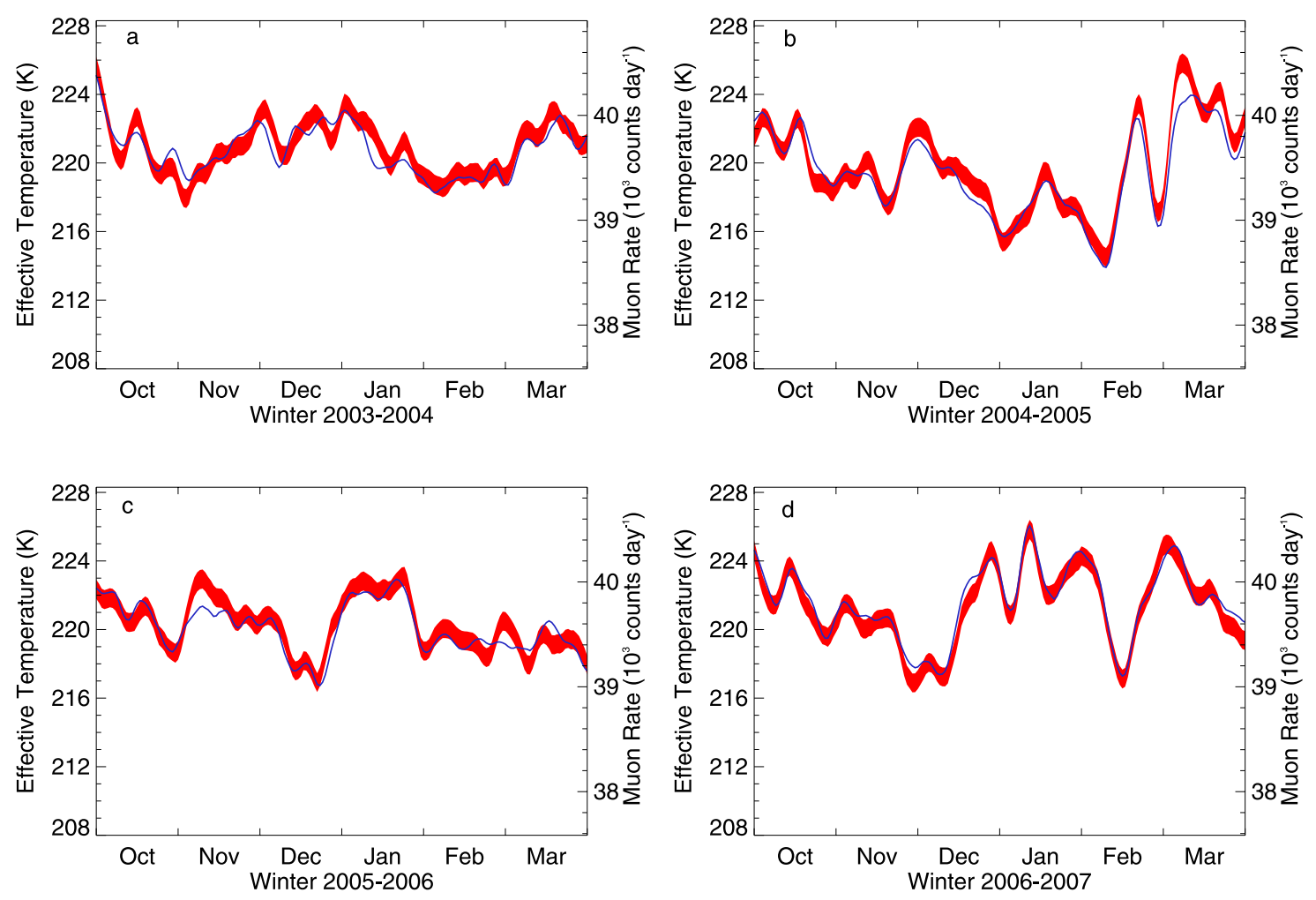

Figure 3. Timeseries of effective temperature for winters 2003-2007 (blue) from ECMWF and daily muon rate \pm 1 standard deviation registered at MINOS Far Detector (red). A five day smoothing has been twice applied to both data. The ticks on the horizontal axis correspond to the start of the labelled month.

future work should include looking for these and related effects in lower energy cosmic muons.

[13] Our results raise the possibility of utilising muon detectors for meteorological applications. Other large underground detectors around the globe should find their data contain similar variability. The positive temperature effect has only previously been seen in variations averaged over seasons [Ambrosio et al., 1997]; here we have shown that much shorter term variations can be seen. Since cosmic rays were discovered in the 1930s, several datasets have been accumulated. During this period, upper-air observations were largely restricted to balloons and were plagued by biases that could change with time. These biases have only recently been significantly reduced. Future studies may show that the early cosmic ray data has captured useful upper-air information which is beneficial to centres such as the ECMWF, particularly as a basis for calibrating long term trends in temperature.

[14] Acknowledgments. This work was supported by the US DOE, the UK STFC, the US NSF, the state and University of Minnesota, the University of Athens, Greece, and Brazil's FAPESP and CNPq. We are grateful to the Minnesota Department of Natural Resources, the crew of the Soudan Underground Laboratory, and the staff of Fermilab for their contribution to this effort. We also acknowledge the BADC and the ECMWF for providing the environmental data for this project.

\section{References}

Adamson, P., et al. (2007), Measurement of the atmospheric muon charge ratio at TeV energies with MINOS, Phys. Rev., D76, 052003, doi:10.1103/PhysRevD.76.052003.

Ambrosio, M., et al. (1997), Seasonal variations in the underground muon intensity as seen by MACRO, Astropart. Phys., 7, 109-124.
Andrews, D. G., J. R. Holton, and C. B. Leovy (1987), Middle Atmosphere Dynamics, Int. Geophys. Ser., vol. 40, 1st ed., Academic, London.

Aplin, K. L., R. G. Harrison, and A. J. Bennett (2005), Effect of the troposphere on surface neutron counter measurements, in Greenhouse Gases, Ozone, and Electrodynamics; Their Changes in the Middle Atmosphere and Lower Thermosphere, Adv. Space Res., vol. 35, pp. 1484-1491.

Barrett, P. H., L. M. Bollinger, G. Cocconi, Y. Eisenberg, and K. Greisen (1952), Interpretation of cosmic-ray measurements far underground, Rev. Mod. Phys., 24, 133-178.

Bazilevskaya, G. A., et al. (2008), Cosmic ray induced ion production in the atmosphere, Space Sci. Rev., 136, 149-173, doi:10.1007/s11214-0089339-y.

Charney, J. G., and P. G. Drazin (1961), Propagation of planetary-scale disturbances from lower into upper atmosphere, J. Geophys. Res., 66, 83-109.

Chen, W., M. Takahashi, and H. F. Graf (2003), Interannual variations of stationary planetary wave activity in the northern winter troposphere and stratosphere and their relations to NAM and SST, J. Geophys. Res., 108(D24), 4797, doi:10.1029/2003JD003834.

Chshyolkova, T., et al. (2007), Polar vortex evolution during Northern Hemisphere winter 2004/05, Ann. Geophys., 25, 1279-1298.

Courtier, P., J. N. Thepaut, and A. Hollingsworth (1994), A strategy for operational implementation of 4D-Var, using an incremental approach, $Q$. J. R. Meteorol. Soc., Part B, 120, 1367-1387.

European Centre for Medium-Range Weather Forecasts (ECMWF) (2007), IFS Documentation CY31r1, II, Data Assimliation, Reading, U. K., (Available at http://www.ecmwf.int/research/ifsdocs/CY31r1)

Feng, W., M. P. Chipperfield, S. Davies, P. von der Gathen, E. Kyro, C. M. Volk, A. Ulanovsky, and G. Belyaev (2007), Large chemical ozone loss in 2004/2005 Arctic winter/spring, Geophys. Res. Lett., 34, L09803, doi:10.1029/2006GL029098.

Gaisser, T. (1990), Cosmic Rays and Particle Physics, Cambridge Univ. Press, Cambridge, U. K.

Holton, J., and H. Tan (1980), The influence of the equatorial Quasi-Biennial Oscillation on the global circulation at $50 \mathrm{mb}$, J. Atmos. Sci., 37, $2200-2208$

Manney, G. L., M. L. Santee, L. Froidevaux, K. Hoppel, N. J. Livesey, and J. W. Waters (2006), EOS MLS observations of ozone loss in the 20042005 Arctic winter, Geophys. Res. Lett., 33, L04802, doi:10.1029/ 2005GL024494. 
Rebel, B. J. (2004), Neutrino-induced muons in the MINOS far detector, Ph.D. thesis, Indiana Univ., Bloomington.

Rex, M., et al. (2006), Arctic winter 2005: Implications for stratospheric ozone loss and climate change, Geophys. Res. Lett., 33, L23808, doi:10.1029/2006GL026731.

Scherhag, R. (1952), Die explosionartigen Stratosphärenerwarmungen des Spätwinters 1951/52, Ber. Dtsch. Wetterdienstes US Zone, 6(38), 51-63.

Sloan, T., and A. W. Wolfendale (2008), Testing the proposed causal link between cosmic rays and cloud cover, Environ. Res. Lett., 3, 024001, doi:10.1088/1748-9326/3/2/024001.

Tilmes, S., R. Müller, A. Engel, M. Rex, and J. M. Russell (2006), Chemical ozone loss in the Arctic and Antarctic stratosphere between 1992 and 2005, Geophys. Res. Lett., 33(20), L20812, doi:10.1029/ 2006GL026925.

Varotsos, C. (2002), The Southern Hemisphere ozone hole split in 2002, Environ. Sci. Pollut. Res., 9, 375-376.

P. Adamson, B. Baller, R. H. Bernstein, G. J. Bock, D. J. Boehnlein, D. Bogert, E. Buckley-Geer, S. Childress, B. C. Choudhary, N. Grossman, D. Harris, R. Hatcher, J. Hylen, C. James, D. Jensen, G. Koizumi, A. Kreymer, P. Lucas, C. D. Moore, J. G. Morfin, R. K. Plunkett, R. A Rameika, B. Rebel, N. Saoulidou, P. Shanahan, W. Smart, A. Wehmann, and R. Zwaska, Fermi National Accelerator Laboratory, Batavia, IL 60510, USA. C. Andreopoulos, A. Belias, C. J. Metelko, T. C. Nicholls, G. F. Pearce, and T. M. Raufer, Rutherford Appleton Laboratory, Chilton OX11 0QX, UK

K. E. Arms, B. R. Becker, E. W. Grashorn, S. M. S. Kasahara, S. Kumaratunga, P. J. Litchfield, M. L. Marshak, J. R. Meier, W. H. Miller, D. A. Petyt, K. Ruddick, B. Speakman, and M. Strait, School of Physics and Astronomy, University of Minnesota-Twin Cities, Minneapolis, MN 55455, USA.

R. Armstrong, C. Bower, M. Ishitsuka, N. Mayer, M. D. Messier, J. Musser, J. Paley, and J. Urheim, Department of Physics, Indiana University, Bloomington, IN 47405, USA.

D. J. Auty, E. Falk, P. G. Harris, J. Hartnell, P. Symes, and M. A. Tavera, Department of Physics and Astronomy, University of Sussex, Falmer BN1 9QH, UK.

D. S. Ayres, M. C. Goodman, A. M. McGowan, J. Reichenbacher, M. C. Sanchez, R. L. Talaga, and J. L. Thron, Argonne National Laboratory, Argonne, IL 60439, USA.

P. D. Barnes Jr., and D. M. Wright, Lawrence Livermore National Laboratory, Livermore, CA 94550, USA.

J. Barnett, G. D. Barr, J. H. Cobb, R. P. Litchfield, S. Osprey, R. Pittam, P. A. Rodrigues, J. Smith, R. Smith, A. Sousa, G. Tinti, A. Weber, and N. West, Department of Physics, University of Oxford, Oxford OX1 3PU, UK. (giles.barr@physics.ox.ac.uk; sosprey@atm.ox.ac.uk)

W. L. Barrett, Physics Department, Western Washington University, Bellingham, WA 98225, USA.
D. Bhattacharya, S. A. Dytman, D. Naples, and V. Paolone, Department of Physics and Astronomy, University of Pittsburgh, Pittsburgh, PA 15260, USA.

M. Bishai, M. Dierckxsens, M. V. Diwan, D. E. Jaffe, B. Viren, and K. Zhang, Brookhaven National Laboratory, Upton, NY 11973, USA.

A. Blake, J. D. Chapman, A. J. Culling, J. S. Marshall, and M. A. Thomson, Cavendish Laboratory, University of Cambridge, Cambridge CB3 0HE, UK.

J. Boehm, S. Cavanaugh, G. J. Feldman, and S. M. Seun, Department of Physics, Harvard University, Cambridge, MA 02138, USA.

D. Cherdack, H. R. Gallagher, T. Kafka, W. A. Mann, W. P. Oliver, J. Schneps, and N. Tagg, Physics Department, Tufts University, Medford, MA 02155, USA.

S. J. Coleman, M. Kordosky, J. K. Nelson, and P. Vahle, Department of Physics, College of William and Mary, Williamsburg, VA 23187, USA.

J. K. de Jong, H. A. Rubin, and C. White, Physics Division, Illinois Institute of Technology, Chicago, IL 60616, USA.

M. Dorman, J. J. Evans, A. Holin, D. J. Koskinen, R. J. Nichol, C. Smith, and J. Thomas, Department of Physics and Astronomy, University College London, London WC1E 6BT, UK.

C. O. Escobar, Instituto de Fsica Gleb Wataghin, Universidade Estadual de Campinas, CP 6165, 13083-970 Campinas, Brazil.

M. V. Frohne and P. Schreiner, Physics Department, Benedictine University, Lisle, IL 60532, USA.

A. Godley, J. J. Kim, J. Ling, S. R. Mishra, A. Rahaman, and C. Rosenfeld, Department of Physics and Astronomy, University of South Carolina, Columbia, SC 29208, USA.

P. Gouffon, Instituto de Fisica, Universidade de Sao Paulo, CP 66318, 05315-970 Sao Paulo, Brazil.

R. Gran and A. Habig, Department of Physics, University of Minnesota at Duluth, Duluth, MN 55812, USA.

K. Grzelak, Faculty of Physics, Warsaw University, PL-00-681 Warsaw, Poland.

A. Himmel, L. Mualem, H. B. Newman, J. P. Ochoa-Ricoux, and C. W. Peck, Lauritsen Laboratory, California Institute of Technology, Pasadena, CA 91125 , USA.

G. M. Irwin, G. Pawloski, S. G. Wojcicki, and T. Yang, Department of Physics, Stanford University, Stanford, CA 94305, USA.

S. Kopp, K. Lang, L. Loiacono, J. Ma, R. Ospanov, and Z. Pavlovic, Department of Physics, University of Texas at Austin, 1 University Station, Austin, TX 78712, USA.

S. Mufson, Department of Astronomy, Indiana University, Bloomington, IN 47405, USA.

P. Stamoulis and G. Tzanakos, Department of Physics, University of Athens, GR-15771 Athens, Greece.

M. Watabe and R. C. Webb, Physics Department, Texas A and M University, College Station, TX 77843, USA. 